\title{
Visualizing Complex Schemas in Description Logic using Movable Lens Filters
}

\author{
Gary K. Ng, Carole A. Goble and Adrian J. West \\ Department of Computer Science \\ University of Manchester \\ Manchester, United Kingdom M13 9PL \\ $\{n g g, c a g, a j w\} @ c s . m a n . a c . u k$
}

\begin{abstract}
In recent years, the distinctions between the requirements and problems in knowledge representation and databases have receded. As the information stored in databases becomes more complex they benefit from the more expressive reasoning and intelligent retrieval techniques offered by knowledge representation techniques. Description Logics, such as GRAIL, can be used as object-based complex database schemas. However, the complexity of visualizing the relationships between concepts in a large Description Logic model is not straightforward; their isKindOf inheritance lattice is inferred on the basis of the composition of the concepts, the legality of various concept compositions can be hard to determine, and frequent patterns of concepts and their relationships are obscured. We have developed a visualization environment based on Movable Lens filters to support GRAIL modelers building a very large medical ontology. Each lens within this environment is effectively a schema browsing component and is associated with a single operation, such as schema querying, filtering by relationships, or attribute visualization. A variety of complex queries and visualization mappings can be constructed flexibly by superimposing the lenses. We describe the design, merits and limitations of this approach.
\end{abstract}

\section{Keywords}

Movable Lens filters, schema visualization, user-level data organisation, description logics, information visualization, visual interface to databases.

\section{INTRODUCTION}

Description Logics (DLs), such as CLASSIC (Borgida et al. 1989) and GRAIL (Rector et al. 1997), are a class of powerful declarative knowledge representation languages based on semantic networks/frames with subsumption and multiple inheritance. Description Logic models have been used in a wide variety of data-oriented applications including the representation of complex schemas for the car industry (Rychtyckyj 1996), software management (Devanbu et al. 1991) and medicine 
(Rector et al. 1997). Description Logics can be seen as a form of expressive compositional object-based schema (Borgida 1995). Recent work concentrates on unifying DLs with conventional DBMS (Baader et al. 1997), combining the expressive modeling and querying power of DLs with the efficient management of large data sets and query optimisation of traditional DBMS.

The development of Description Logic models is labor intensive and iterative. The inspection of the properties and relationships of objects (or concepts, in DL terms) is one of the major tasks in model development.

The inspection is required for two major reasons:

- for verifying the consistency and soundness of concept properties and relationships in order to ensure that the modelers have introduced concepts and relationships at the correct level of abstraction,

- to survey and identify reusable modeling constructs and patterns for modeling similar phenomena.

DLs differ from more conventional data models in two ways. Firstly, many of the relationships between concepts are inferred and hence implicit in the model, unlike conventional data models whose relationships are explicitly asserted. In particular the subsumption or isKindOf relationship, usually asserted as an inheritance hierarchy in object-based models is implicit, and automatically inferable in a DL based on the concept definitions. Secondly, the combination of concepts to form new compositional and complex concepts is controlled. The impact of these controls, and consequently the concepts that are permitted or excluded, and hence the coherency of the model, are often also implicit.

The common method to verify the correctness of the model is by repeatedly asking general questions about various subsets of objects, visualizing and comparing their relationships. These issues are particularly pressing for a large and complex model such as the medical terminology model implemented in GRAIL (Rector et al. 1993). The model is being used as a schema mediator between clinical information systems of different countries and languages, currently consisting of around 8000 elementary objects, 600 different types of relationships, and around 18000 explicit relationships.

Conventional textual browsing tools are helpful when the modeler is focusing on one concept, but are poor when the modeler is required to examine the interactions and patterns between multiple concepts. The size of the model means that the modeler can quickly lose their way and miss an overall perspective of sections of the model if they are only able to interact on a concept by concept basis. A tool capable of presenting views on clusters of concepts where these clusters are selectable by the user, would be desirable as the inspection tasks demands flexible manipulation and interaction with various collections of conceptual data, as well as a variety of visualization perspectives appropriate to the inspection tasks.

Motivated originally to aid the inspection tasks of the GRAIL medical terminology model, we set out to provide an uniform, flexible and integrated visualization environment for such data and tasks. Our tool is based on the use of movable lens filters 
(Bier et al. 1993) as units for query, data manipulation and visualization. Lenses are a new class of user-interface tools (Bier et al. 1994) that combine an arbitrarily-shaped region with an operator that changes the appearance of objects viewed through that region. The key features of our proposed tool are:

1. Data interaction: The ability to map and filter abstract data by queries using lenses. Useful items can be extracted and retained from lenses, either as examples for modeling or as items for further inspection. Items can be organized spatially in a desktop like environment.

2. Visualization: Using combinations of lenses to explore and inspect the content of existing models, identifying useful sub-parts in the form of graphical patterns.

3. Composition of tools: Constructing complex queries and visualization mappings by superimposing lenses to form stacks, contrasting to the data-flow network approach employed in other visualization systems.

Lens have been applied in the past to numerical data; here we apply them to abstract data, i.e. concepts and relationships in a DL model or schema, and not to the data instances that the concepts might describe.

After related work, a brief introduction to the GRAIL formalism is given. The proposed design of the lens system is then described, within the context of model inspection scenarios and the problems associated with the textual browser. The solutions using lenses are then illustrated with our prototype implementation, followed by a discussion of the approach and our plan for further development.

\section{RELATED WORK}

Scientific Visualization systems such as AVS, Khoros and Data Explorer (for a review, see (Brodlie et al. 1995)) allow the construction of complex data transformations, filtering, and visualization mappings by visually linking $\mathrm{I} / \mathrm{O}$ compatible modules of various functions into a dataflow network diagram, with boxes representing functional modules and the links between boxes specifying the direction of data flow. Such systems deal primarily with data which has a strong ordinal nature, such as numerical data. The specification, filtering, and cropping for subsets of data are achieved with functional modules such as range boundary setting and thresholding.

The methods for ordinal data manipulation such as filtering and cropping do not apply to semantic knowledge models which contain only abstract symbolic (or nominal) relational data. The manipulation of subsets of data in knowledge models relies heavily on queries. Unlike other systems in which queries are only performed once at the start of the visualization process to specify the data to be visualized, in our problem queries are required to filter the data to form various collections, as well as to determine how a data item is mapped onto visual attributes such as colors and shapes.

Clearly a variety of filtering and visualization modules with the capability of querying a database could be devised within such systems. Complex queries could 
be formed by linking the modules in a variety of ways, specifying the flow of query results. However, the visualization of results are usually at the end of a complex query, whereas our model inspection tasks are iterative and demand a continual visualization of results at each step.

The combination of layered filters and movable viewing lenses as see-through graphics editing tools was first proposed in (Bier et al. 1993, Stone et al. 1994). A variety of click through lenses mounted on a transparent layer were employed in a two handed interface. The intended benefits were to save steps in selecting editing operations relative to traditional menu interactors. Lenses were used to provide alternative views of graphical objects, by filtering various visual attributes, changing colors or line styles, or modifying their order of rendering, local to regions of interest.

The use of lenses as a means to perform dynamic queries (Ahlberg et al. 1992) to a database was first applied to census data (Fishkin and Stone 1995). The lenses operate over a geographical plot of the data. Each lens is associated with a dynamic slider, filtering data of interest according to a numerical threshold. Complex queries can be performed by superimposing the lenses, and conjunctive and disjunctive combinations can be specified with the controls on the lenses.

Each lens in our environment is a module with a specific function. Our system saves the steps in linking up modules explicitly by constructing complex queries and visualization mappings with superimposed lenses to form stacks, with the trade-off of visual occlusion of previously laid down modules. Our tool extends the ideas in (Fishkin and Stone 1995) on several fronts:

1. Applying the lens paradigm to non-spatial abstract data.

2. Different data filtering and cropping functions appropriate for semantic data types. Each lenses stack is effectively a placeholder for various collections of data.

3. Employing lenses not only as a component for threshold filtering or zooming on a given set of data over a fixed visual representation, but also as a dataflow programming construct for visualization. Various subsets of data could be channelled into a single visualization or further filtering operations. Alternatively, a single set of data could be branched to different operations.

\section{DESCRIPTION LOGICS AND GRAIL}

A brief explanation of the salient characteristics of Description Logics generally, and GRAIL in particular, is necessary in order to understand the schemas to be visualized and the tasks undertaken by the domain modelers. For an overview of Description Logics see (Borgida 1995), for more details on GRAIL see (Rector et al. 1997).

DLs are a family of logics designed to represent the taxonomic and conceptual knowledge of a particular application domain on an abstract level. Their basic building blocks are concepts, roles and instances (called individuals). A concept denotes (describes) a collection of instances, and roles denote binary relationships between instances. Recursive concept constructors associate a base concept with a set of roleconcept pairs; the concept that fills the role is known as the role filler; in GRAIL 
the concept constructor is called which. A concept that is constructed from a base concept and role-filler pairs is known as a defined concept, and can be given a name. For example three defined concepts could be TraumaLocation: Trauma which <hasLocation-Bone>, TraumaCondition: Trauma which <hasConditionsevere>, SevereBoneTrauma: Trauma which <hasLocation-Bone, hasCondition-severe>. Role filler concepts or base concepts can be defined or primitive. There are two key aspects of the GRAIL Description Logic.

\subsection{Automatic classification}

Concepts define a subsumption (or isKindOf) lattice. A primitive atomic concept has no characterising roles applied to it, and is placed in the subsumption hierarchy by the system designer, for example, Fracture isKind0f Trauma, Bone isKindOf BodyPart, Femur isKindOf Bone. Defined composite concepts are placed in the hierarchy automatically by a classifier based on their definition. For example, FracturedSeverefemur: Fracture which <hasLocationFemur, hasCondition-severe $>$ is classified automatically as a kind of Trauma Location, TraumaCondition, SevereBoneTrauma, Fracture and Trauma. The instances of the concept FracturedSevereFemur are said to be included in the instances of concept Fracture. So the fundamental difference between DL and traditional knowledge representations is that while hand-crafted assertion is the main modeling act in traditional systems, DL automatically assimilate new concepts into a taxonomy by classifying them with respect to the concepts that are currently in the concept taxonomy and adding appropriate isKindOf relationships. Once classified, the new concept will inherit the descriptive properties from all its parents.

\subsection{Composition Control}

The combination of concepts by roles is tightly controlled. GRAIL differs from other DLs in this respect in that it has role sanctioning. A sanction is an assertion that two concepts, and hence all their descents, may be related via some role. Compositions of concepts cannot be formed unless an appropriate sanction on the role is present. In GRAIL there are two levels of role sanctioning: grammatical role sanctions express general relationships between concepts and sensible role sanctions further elaborates a grammatical sanction to ensure that only more specific semantically valid compositions are formed. For example, we may assert that <Trauma hasLocation-BodyPart> is grammatically sanctioned, permitting the definition of Fracture which <hasLocation-Femur>, but also Fracture which <has Location-Tongue $>$. $\langle$ Fracture hasLocation-Bone $>$ is sensibly sanctioned statement indicates that Fractures occur in bones so it is not permissible to define the concept Fracture which <hasLocation-Tongue>, but it is permissible to create and classify the concept FracturedBone: Fracture which <hasLocation- 
Bone>. It doesn't mean, however, that FracturedBone has been created and classified, only that it could be. The levels of sanctioning are hierarchical and provide flexible control over the formation of compositional concepts.

Sanctioned roles mean that we can pose the question: "what can I say about a concept?". We see what sanctions apply to the concept, and return an answer based on these. This means that we can generate new concepts that are implied by the taxonomy from a concept and its sanctioned roles so new composite concepts can be constructed by applying sanctioned roles to base concepts. GRAIL modelers capitalize on the fact that the existence of composite concepts are implied by the taxonomy and can be readily generated to build small and sparse but elegant models. Hence many of the concepts in the GRAIL subsumption lattice may not actually explicitly exist but they are implied, can be created and classified as they are asked for and be positioned in lattice with respect to the concepts that are there at that point in time. For example, the sanctions mean that concept FracturedBone is possible but it does not explicitly appear in the classification lattice described above until it is asked to be classified. FracturedBone would be a direct child of Fracture and SevereBoneTrauma and a direct parent of FracturedSevereFemur if it were classified. Hence the taxonomy in GRAIL is built dynamically.

\subsection{Problems with Modeling}

If a role is asserted between two concepts too low in the hierarchy, then concepts above them that would make sense to define and classify using that role are forbidden. The model is over-constrained and its generative capability is inadequate. An under-constrained model has roles asserted too high up the hierarchy so that nonsensical concepts are permitted to be defined or generated. For example, asserting that Trauma (sensible) hasLocation BodyPart is too general, allowing nonsensical concepts such as fractured livers to be permissible; Trauma (sensible) hasLocation Bone is too restrictive, excluding traumas to any other organ of the body. The problem is that the modeler cannot see the consequences of sanctioning a role because the concepts subsequently implied are implicit in the model, and only arise when they are defined and classified or explicitly generated.

Large and long lived models are built incrementally, and most often involve teams of modelers. It is therefore necessary for the modelers to be able to survey a model that they have forgotten the details of, or developed by other modelers. A misplaced sanction could lead to unexpected classifications of defined concepts in the future, either because some isKindOf relationships are not implicable and therefore not generated, i.e. missing parents or decendents; or some spurious isKindOf relationships have been created, i.e. extra parents or decendents. The method to verify the correctness of classification is to repeatedly inspect the model by ask general questions about concepts. 


\subsection{Model Inspection}

A modeler inspects the GRAIL model through interaction with a GRAIL Terminology Server (TeS)(Bechhofer et al. 1997). The TeS provides operations relating to the content of the conceptual model including those for inspecting:

- the model hierarchy by obtaining the subclasses or superclasses of a single concept, obtaining all the descents of a single concept or testing whether a concept is a descendent of another;

- the properties of a concept in order to determine how concepts and roles can be combined to create new legal composite concepts, by obtaining the grammatical and sensible roles for a single concept.

The current inspection process is oriented around inspecting the model concept by concept, which is adequate for navigating around the model, and to present a concept's properties for investigating how they can be combined to form new ones. However, there is a gap between the services provided and the questions asked by modelers for the purposes of inspection and surveying collections of concepts and roles simultaneously.

\section{(a) Verification}

The classification links defined concepts to many different parts of the model. To ensure the correct classifications of a defined concept with respect to the modeling domain, the modelers need to verify that a classified concept meets certain expectations in its linkages to a particular set of parents and/or decendents. For example, the decendents of the defined concept AnatomicalSystem should all have a role hasStructuralComponent, and the role filler being a decendent of BodyPart.

A function which obtains automatically all the roles for each of the immediate decendents of AnatomicalStructure could be useful. Collectively as a set of roles, the visual aid of highlighting could then be used to bring out all the hasStructural Component roles, and then highlight those roles with a filler which is a BodyPart.

\section{(b) Surveying for Patterns}

In large and complex models, the same patterns between concepts and role often repeatedly appear across a set of concepts (primitive or defined) located in different parts of the hierarchy. These patterns could be reused:

- as a reminder collection of roles which may need to be sanctioned or specialized when defining a new concept which is similar in essence, or

- to ensure that a specification of a new defined concept with the collection of identified roles will result in an expected classification, so as to minimize the need for verification. 
For example, the primitive concepts Heart, Lung, and Stomach, which are not defined under the same set of parents within the model, will have a common set of roles for describing their function, their linkages to blood vessels, and linkages to Diseases...etc. Identifying such set of roles as reference could be beneficial when other organs such as Kidney is defined, especially if the modeler working on Kidney is not the same person who was working on the others.

Again, an automatic function to obtain all the roles for each of the concepts Heart, Lung, and Stomach would be useful. In order to identify a common set of roles, the visual aid of aligning the roles of different concepts uniquely across a form of a matrix could be used, such that any commonalities or clustering could be seen as a synopsis. It would also be helpful if the set of pattern could be selected and saved for future reference.

To summarize, our requirements are:

- A function which obtains all the relevant information for a collection of concepts automatically;

- A place to hold an arbitrary collection as inputs, and a place to store a collection of results;

- Highlighting items of particular interests from a collection;

- Items in a collection should be able to be aligned to survey clusters and trends across items;

- Some form of storage for an arbitrary selected list of items.

\section{THE BASICS OF A GRAIL LENS}

In this section, the fundamental concepts of our lenses, and the platform on which our prototype system is based on are introduced. The particulars of our lens design with examples of use are illustrated in detail in the next section.

\subsection{PadDraw Surface and Lens}

The system currently under development is based on a paint program called PadDraw, an application of the zoomable graphical interface widget Pad++ (Bederson et al. 1996). PadDraw provides a graphical surface that can be panned and zoomed. Many graphical primitives can exist on this surface, including lines, polygons and text. Primitives are layered and can be resized, scaled and dragged. They can be entered interactively by the user or generated dynamically from within Pad++. Primitives can be placed at any location and at any scale, allowing the efficient use of space.

PadDraw is used to implement the notion of lenses, where a lens is a region that shows a modified view of the surface that the lens is positioned over, just as a magifying lens magnifies a surface. Lens can be layered on top of one another; each modifying the view of the one beneath. Figure 1 is a demonstration of PadDraw 
showing a table of numbers on the surface (Figure 1a), and a lens covering the same table of numbers (Figure lb). The visual representation is changed from a table to a graph as viewed through the lens.

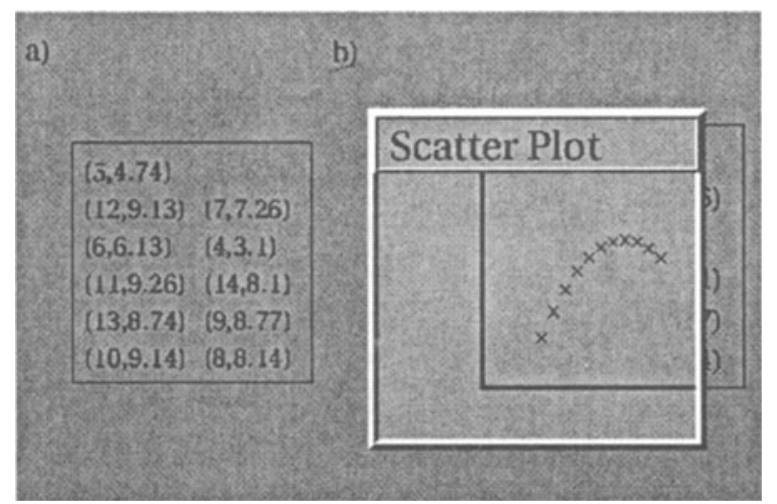

Figure 1 PadDraw: a) A table of numbers and b) A scatter plot Lens over the table of numbers.

\subsection{Lenses for Description Logic}

The PadDraw surface could be used as a place to specify an arbitrary collection of concepts, as an entry point to the model inspection process, in the form of textual primitives. Lenses, while being a bounded region graphically on a freely drawable surface, are layered and can associate with an operation to apply to objects which it covers. Lenses can be used in our problem to:

- Bound a collection of items from the surface as the focus for inspection.

- Apply its operator to the collection one by one, the operator would be the automatic function to obtain information from the TeS.

- Store the results as a temporary collection.

- Present the results graphically on the lens itself, binding the results to the operator and input, providing a visual layout to aid the comprehension of results.

- Highlights the input items by applying the operator to the collection and use the result to change the colors of the items of interest.

Functionally each lens has a set of input data and a set of output results, and an operator to map its inputs to output. All data primitives (more detail later) covered by a lens's main area are regarded in its input set. Changes in the operator and/or the input set (by moving the lens or the items underneath) result in a recalculation of the output set. In this way, it is possible to think of a lens as a partial function. 
The Pad++ widget is modified to act as the interface front-end for the GRAIL terminology server. Figure 2 shows the visual design of a lens. The operator which governs how the inputs are mapped to its output is specified in the properties panel of the lens. The display of the panel is triggered by double clicking on the flap. The position of the flap with respect to the lens' main area acts as a visual element for distinguishing between different types of lenses. Coincidentally, we have developed four lenses, the flap is attached to a different edge of the main area for each type of lens.

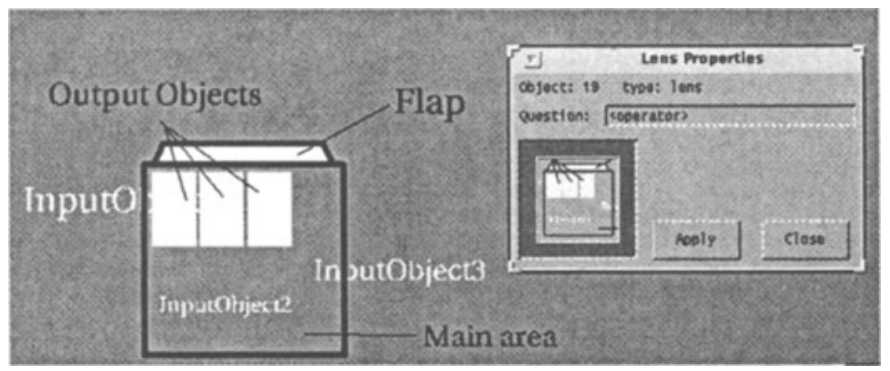

Figure 2 A Grail lens. Results are displayed as tiles. Normally the tiles fill the space available in the lens' main area.

The operator is applied to each input in the set in turn to generate the result. The specification of the operator is in the form of a simple query language. The language allows TeS services and set membership testing to be specified. For example:

- to obtain the set of parents/decendents of a concept (set-generating),

- to obtain all roles of a concept (set-generating),

- to test whether an item is a kind of another item (boolean),

- to test whether an item is a member of a set (boolean).

The mapping between each input and output can be one to one or one to many, depending on the specification. Currently in our design, the presentation of outputs are only as rectangular tiles in the main area, one per result item. The layout algorithm of the result items depends on the type of lens.

Alternatively the result could be used to modify the visual attributes of the original input, such as its color. By default, each output item has the color of the corresponding input item.

\subsection{Representation of Data and I/O between Lenses}

Our lenses regard two types of object as potential inputs: text primitives on the PadDraw surface, or individual tiles as displayed in the main area of a lens. A lens can cover a number of a lenses and text primitives simultaneously. All (fully or partially) 
covered primitives or tiles are included into the lens' input set. A single lens can also be covered by many other small lenses. Thus each small lens may have a different input, depending on its location with respect to the larger one.

Each data item represents either a concept or a role. A concept is simply a string whereas a role is an array of 4 string elements: the base concept, the role, the sanction level, and the role filler. The content of a data item is revealed in a "bubble help" box when the mouse pointer is placed above it.

Questions or collections are constructed incrementally using a stack of lenses. The results are updated automatically, triggered by changes in either the input collection or the operator function. For example, the lens has moved, its input objects have moved out of bounds, or a new function has been specified. Changes are propagated from the bottom to the top of the stack. In effect, the lenses work like dataflow programming artifacts.

\subsection{The GRAIL-Lens}

Our approach breaks down the iterative inspection process as consisting of four basic types of recurring operations: mapping, visualization, filtering and collections. These operations are linked in a variety of ways to form complex functions. We have developed lenses that implement each of these operations:

A Mapper lens associates with a set-generating operator. It takes only concepts as input, ignoring roles, and displays the resulting concept or roles within the boundary of the main area as tiles, one tile per result. The lens displays the results as a matrix. Each input concept is mapped to one row; each result is mapped to a column; the columns are unordered.

A Visualizer lens takes only roles as input, ignoring concepts, and redisplays the inputs within the boundary of the main area as square tiles, one tile per result. The Visualizer lens does not interact with the TeS. It rearranges an input collection of array items graphically, and presents them in a aligned matrix as its results. The information content of the input and output collection are identical except that item's physical location may appear to be different. The collection of results are plotted, in a matrix with unique base concepts as rows, and unique role names as columns.

A Highlighter lens associates its boolean operator with a color. It takes either concepts or roles as input. It performs the logical operation with each object in the collection in turn. The object's color is changed to that associated with the lens if the result is true.

A Collector lens. The Collector lens is designed for collecting an arbitrary collection of item as reference or use as another starting point of inspection. Any items from the surface or the lenses can be collected onto the Collector lens by first covering the item and then click on that item through the Collector lens. The Collector can then be used as inputs to other lenses, or simply occupying space on the surface as a reminder. The details of a collection can be viewed as a list through its control panel. 
Two of the lenses (Mapper and Highlighter) interact with the TeS through the query language, the other two lenses are merely used for manipulating the results. Two of the lenses (Mapper and Collector) are set-generating, and the others are transforming the presentation of the inputs.

The lenses are used to support the verification and surveying activities discussed earlier, and in the next section we give two case studies of their use.

\section{THE GRAIL LENS IN USE}

\subsection{Verification Problem}

Consider the task to verify the modeler's hypothesis "All concepts which are the decendents of the concept AnatomicalSystem have at least one role with the relation hasStructuralComponent, and that the role filling concept within each of these roles are decendents of the concept BodyPart".

Fundamentally the task is testing the membership of a collection of concepts' properties (role fillers of hasStructural Component) within another collection (decendents of BodyPart. For this task we may use a combination of Mapper and Highlighter lens.

\section{(a) The Mapper Lens}

On a graphical surface, multiple concepts could first be specified as textual objects as the entry point concepts. A Mapper lens has a GRAIL TeS query associated with it, which is its operator. As the lens covers objects on the surface, those at least partially covered are considered to be the lens's input collection. The output of a mapper lens will be concepts or roles, that can in turn be the input to another lens. A Mapper with the query "obtain all decendents" covering the text object AnatomicalSystem could be used as our starting point.

\section{(b) The Highlighter Lens}

The task could be made easier by automatically highlighting those items that are of current interest using colors. If the item is a role, we select a single element on which the operator will apply. In our scenario, a Highlighter lens with the operator "role name $==$ 'hasStructuralComponent"' substitutes the role name of each input role from its input collection and applies the logical operator. Those objects which satisfy the operator are highlighted, so we get all the 'hasStructuralComponent' roles highlighted.

The boolean operator can include set-generating queries to the terminology server. This differs from the Mapper in that the set is not treated as the result but used internally to derived the boolean result. Such operator is useful in situations where set membership tests are beneficial. For example, the last step of the verification task in our scenario; to verify that the role filler of all roles are decendents of BodyPart. 
To return to our question, we could type AnatomicalSystem onto the surface as a text object, and then use:

1. A Mapper lens (A) covering the text to generate all decendents of AnatomicalSystem (Figure 3).

2. Define a Mapper lens (B) with the query "obtain all roles". Use (B) to cover the whole of (A) to generate all roles for each of the result in (A) (Figure 4).

3. Define a Highlighter lens (C), and covering (B) (Figure 5) to highlight those roles that are hasStructuralComponent (rolename $==$ 'hasStructuralComponent').

4. A Highlighter lens (D) covering (C) to highlight those roles with a filler that is a decendent of BodyPart ("role filler ISKINDOF 'BodyPart") (Figure 6).

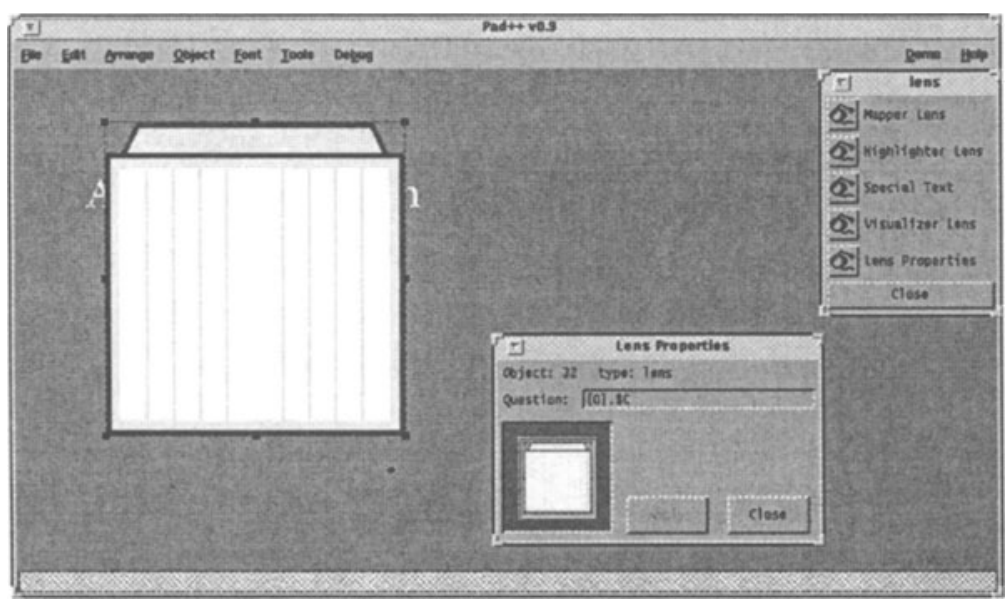

Figure 3 A Mapper lens covering a text object "AnatomicalSystem". The lens operator is specified to obtain all subclasses (Currently this is specified as a element-selector.function-code pair. Element selector treats each input item as an array of strings ([0] is therefore the first element). There are 10 result tiles, filling the space avaiable on the lens.

The highlighted results in (3) and (4) should be identical, otherwise the modelers should realise there is an inconsistency in the model.

\subsection{Surveying for Patterns}

Consider the task of identifying "A collection of role relations which are common across concepts Heart, Lung, and Stomach"

Whilst the Highlighter lens provides visual aid by identifying objects with a specific test criteria, such as in the previous case testing the relationship name against 


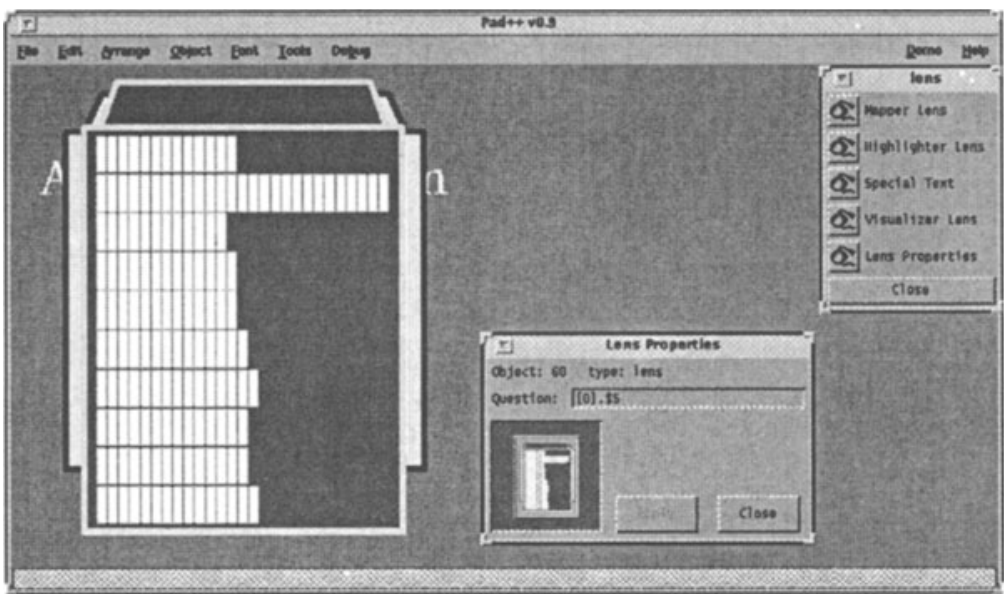

Figure 4 A Mapper lens with an operator to obtain all roles. It is covering the results from Figure 3. The results is 10 rows (one per input), each row showing the roles of an input item.

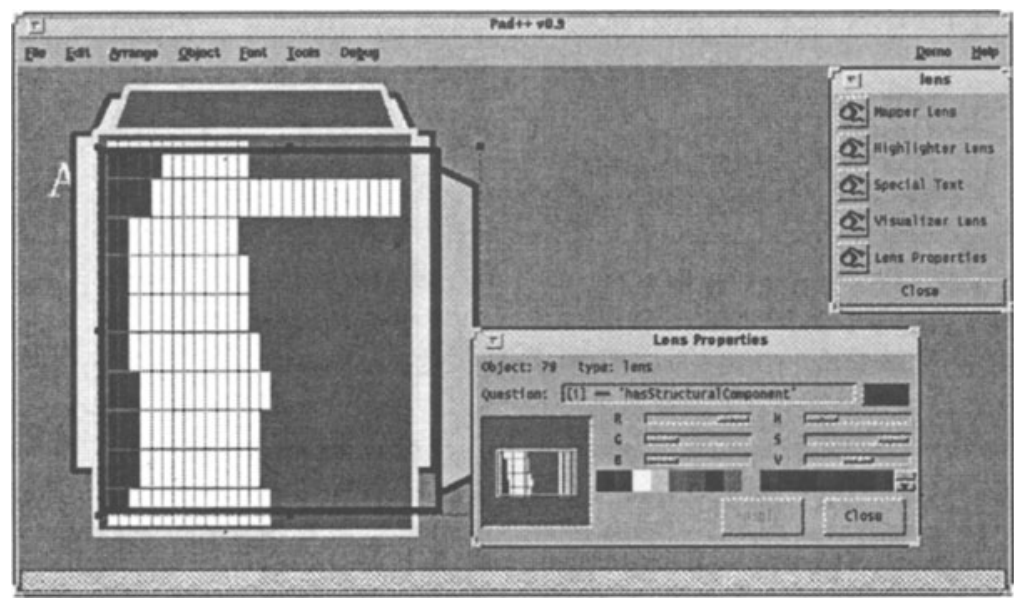

Figure 5 A Highlighter lens with the operator "role name == 'hasStructuralComponent", with red as the highlight color ([1] selects the role name). It is covering the Mapper lens from Figure 4. Roles on the Mapper satisfying the logical operator are highlighted

hasStructuralComponent, the commonness of an arbitrary collection of symbols does not lend itself to a specific criteria for testing. There can be many degrees of commonness within the collection and there could be many subgroups and clusters. To solve this request we use a combination of the Mapper and Visualizer. 


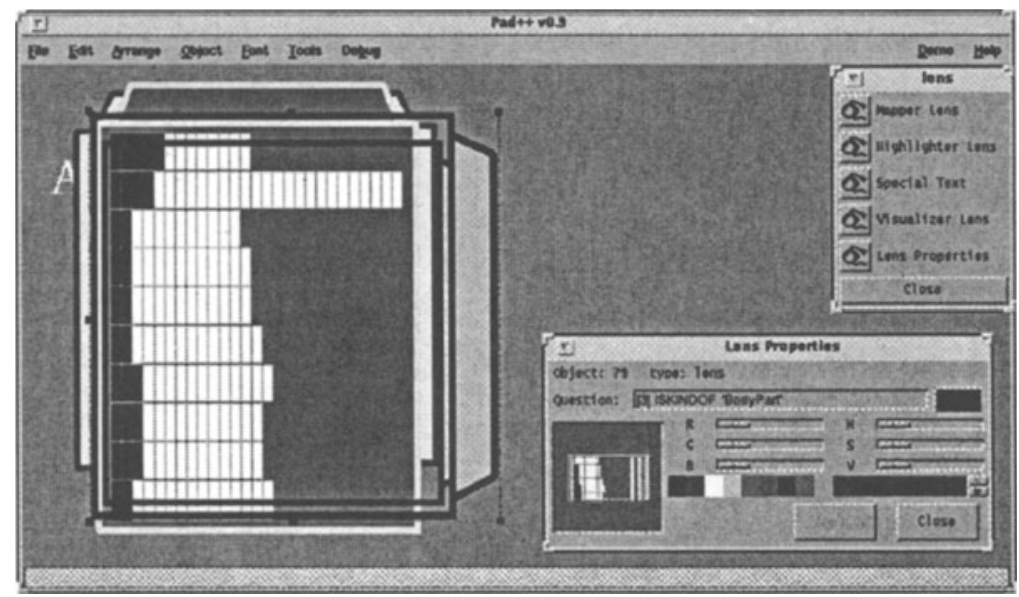

Figure 6 Another Highlighter lens with the operator "role filler is a kind of 'BodyPart", with black as the highlight color ([3] selects role filler). Satisfying roles are highlighted.

\section{(a) The Visualizer Lens}

The alignment calculation is based on the uniqueness of names amongst items. For example, the alignment mapping "row=base concept col=role name" shows that each row represents a unique base concept and each column represents a unique relationship name. The role item Heart sensibly isServedBy CoronaryArtery would appear at the conjunction where the column represents Heart and row represents isServedBy.

There are only 2 dimensions on this display, but each role item has four "attributes" in the array. Therefore several different mappings are possible and some locations may have more than one item. The control panel allows the mapping of different item "attributes" to rows and columns.

\section{(b) The Collector Lens}

While most collections of semantic data can be obtained from conjunctive and disjunctive queries, such as to obtain all roles for concepts, an arbitrary collection of items can only be specified via textual input or direct manipulation from a larger set on screen. There are two implications. Firstly, extraction from result collections forms yet another collection. Secondly, the gathered collection should possess the same behaviour as the queries generated ones.

Although not currently implemented in our prototype. The Collector lens is designed for this purpose. Any items from the surface or lenses can be collected onto the Collector lens by clicking on that item through the Collector lens. The Collector can then be used as inputs to other lenses, or simply occupying space on the surface as a reminder. The details of a collection can be viewed as a list through its control panel (Figure 7). 


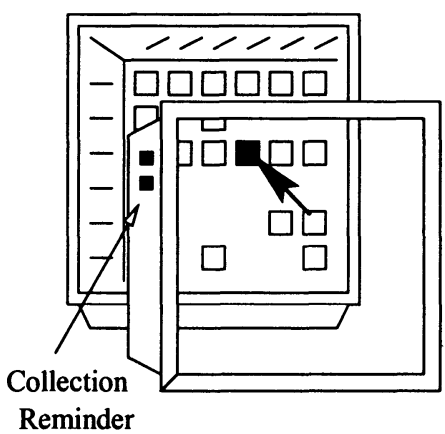

User's head on view

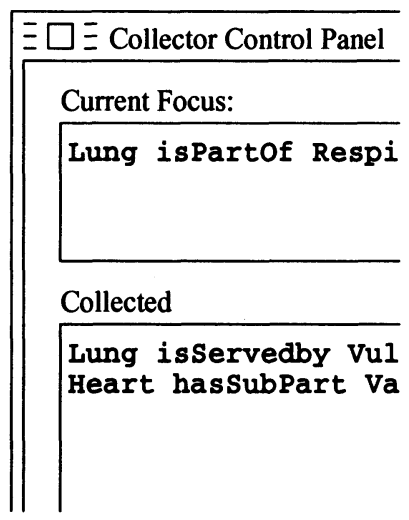

Figure 7 A Collector Lens. Items are collected by clicking on them through the lens. The collection is listed in the property panel.

Therefore, to solve our survey problem we type Heart, Lung, and Stomach as text objects on the surface (Figure 8) and use:

1. A Mapper lens (A) to generate all roles for each of Heart, Lung, and Stomach (Figure 9).

2. A Visualizer lens (B) covering the whole of (A) to re-align all the roles such that unique base concepts are down the vertical axis and unique role names across the horizontal axis, and plot the collection of roles according to the alignment (Figure 10).

3. A Collector lens (C) covering the whole of (B) and let the user select whichever item to be saved as an arbitrary collection.

\section{DISCUSSION}

The four types of lens can be classified based on two of Bier's (Bier et al. 1994) axes for see through tools: Output transparency and operation class. Output transparency describes the appearance of the lenses. Our lenses are either completely transparent (Highlighter and Collector), or occluded by data items (Mapper and Visualizer). Operation class describes the functional property of the lens with respect to the rendering of the graphical primitives. Our lenses are either modifying the color of the primitives (Highlighter), transforming their positions (Visualizer), or transforming by mapping an item to a different set of items (Mapper). There is another axis under operation class that is unique to our lenses. A lens' operation can either lead to queries to the terminology server (Mapper and Highlighter), or the collection of input items alone contain sufficient information to complete the function (Visualizer and Collector).

Initial preliminary evaluation by GRAIL modelers are promising. The ability to 


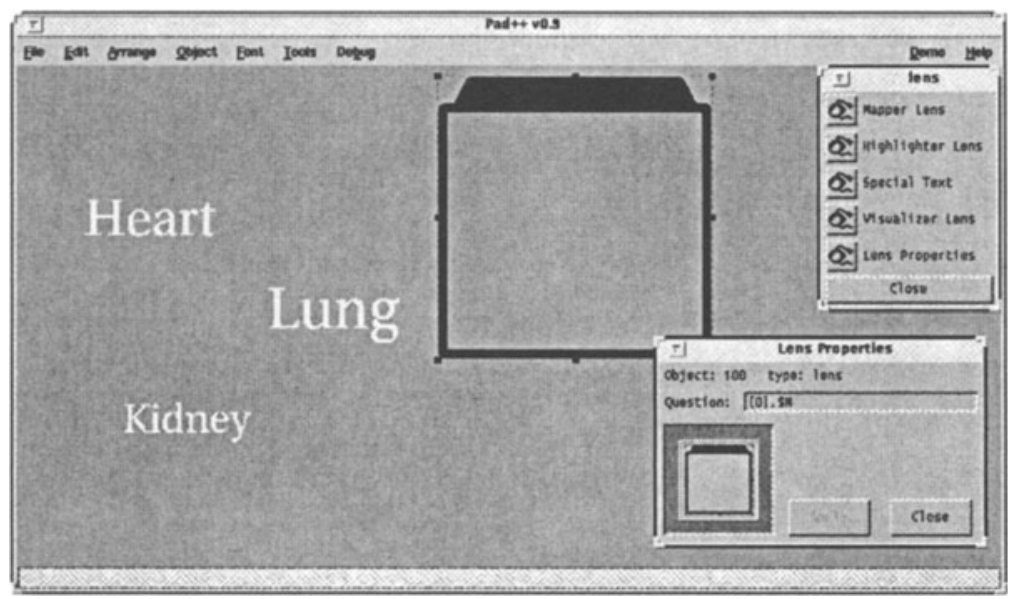

Figure 8 Textual objects Heart, Lung and Kidney with a Mapper showing all roles being defined.

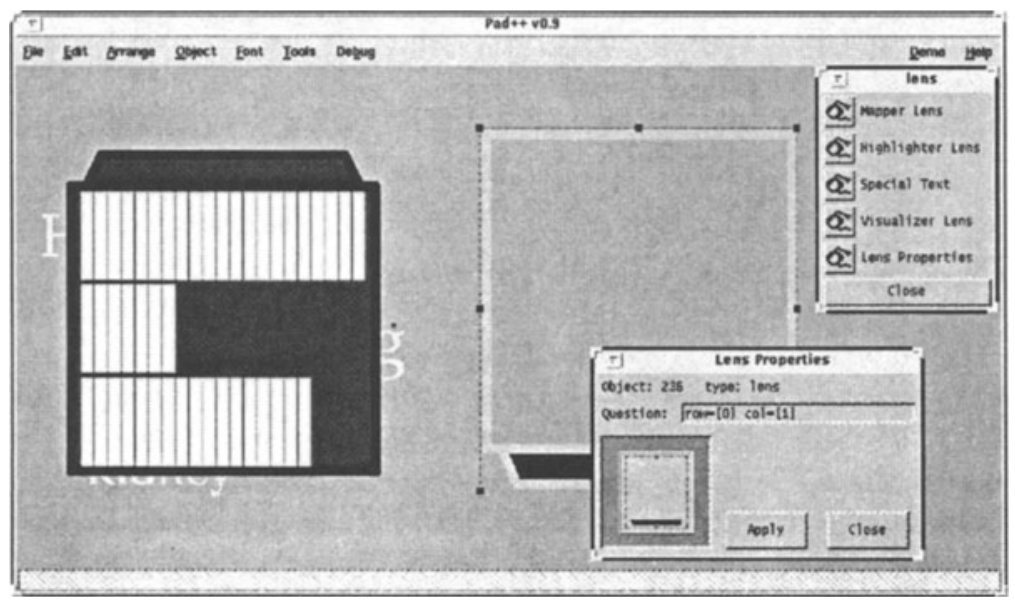

Figure 9 The Mapper is covering the 3 textual objects and generated roles for each. On the right hand side, a Visualizer lens is being defined.

combine simple modules in a variety of ways to form complex visualizations, manipulations and the extensibility are seen as powerful features. Some concerns expressed are discussed in this section.

Style of Interaction With this interface it is necessary that items for investigation must first be gathered spatially in order to be manipulated, which includes initially placing the items onto the surface. This is an extra task compared to dynamic queries which allow the selection of items of interest via sliders.

The current lens designs are intended to be elementary. As a result, usually many 


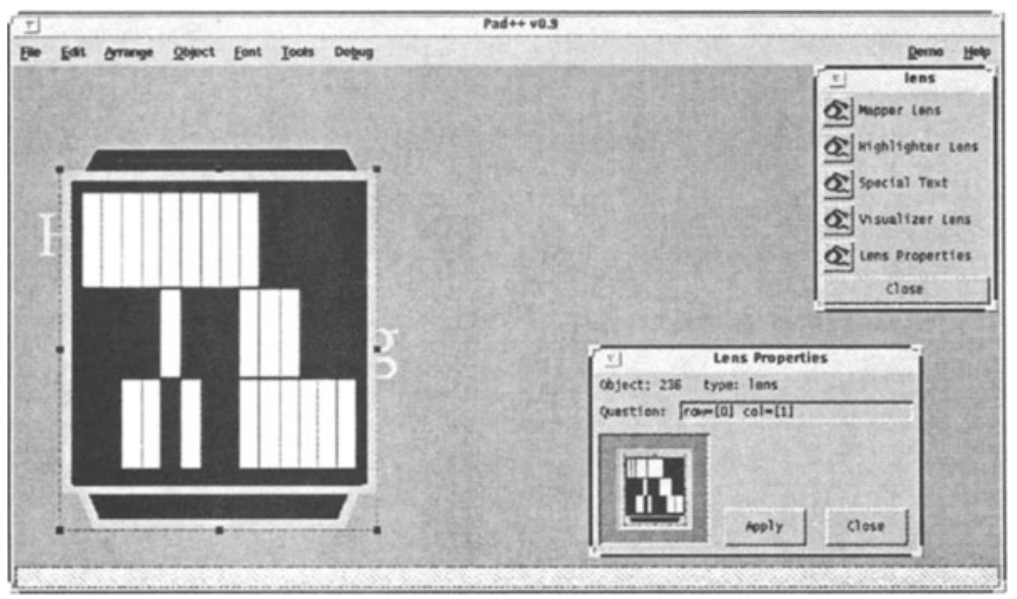

Figure 10 A Visualizer lens showing an aligned matrix of unique concept by unique roles. The result is a matrix of roles where each row represents a base concept and those with common role names are in the same column. Blank entries represent the absence of a role involving the corresponding concept-role pair.

lenses are required to perform something useful. More macro lenses with compound functions will increase their ease of use. We have not given much attention in its investigation at this preliminary stage. The semantics of lenses stacks and combinations are not yet fully defined. The current design allows incompatible queries to be combined, but incompatible lenses' I/O are simply ignored.

Lenses stack allows data to be passed from one to another only upon overlapping. It is a disadvantage as previously laid down modules are occluded and only a limited amount of linkages is possible without compromising the visual clarity. We intend to use the feature of Portals of Pad++ to address this. A portal is a bounded region through which a remote part of the PadDraw surface can be interacted with. It allows references of locations to be made. Portals can be used to branch our lens output to other lenses at other locations.

Functionality The set of queries allowed at the moment are limited. The language currently used for specifying lens operations covers a small set of TeS services and logical operations on set membership. A fuller set meeting the real demands of modelers is expected to be derived during usability tests.

Visual Representation As we cannot predict when and where the user would like to extract items of interest, collections of results from each TeS interaction and at each stage of the inspection process shall be flexibly presented, which is in favor of the use of lenses for the visualization of such data.

Traditional graphical tools for presenting static semantic knowledge are either based on indented text or connection diagrams (node-link) (Fairchild et al. 1988, Patel and Sutcliffe 1993, Dodson et al. 1995, Paley et al. 1996). While connection 
diagrams are widely used and proven valuable, especially for illustrating flow or event related processes (such as inference in (Patel and Sutcliffe 1993, Dodson et al. 1995)), or displaying and editing a reasonably small amount of static inter-related knowledge structures. For tasks such as to identify trends and clusters of relationships, and to select arbitrary items of interest from the representation flexibly, we believe the connection diagram is inferior to more simple visual forms such as those well established techniques for statistical data: scatter plots and reorderable matrices (Bertin 1981). Our design was based on the compact colored square-tiles idea such as seen in recent systems: VisDB (Keim and Kriegel 1994), Attribute explorer (Tweedie et al. 1996) and IVEE(Ahlberg and Wistrand 1995).

The visual representations currently provided are primitive and are devised as a proof of concept demonstration. There are prospects in their improvements with other visual representations. For example, glyphs can be used instead of square tiles. Glyph is a complex graphical object capable of representing a number of attributes simultaneously. Its shape is constructed from a number of graphical primitives and each primitive is mapped to a certain attribute of the data.

From the screen dumps in previous sections, the stack of lenses, their input and output together are rather confusing visually. Visual cues including depth cue and relief borders may increase their readability. A combination of data-flow network and lenses stacks is worth further investigation. For example, a side view of lenses stacks may relieve the visual occlusion problem of stacks of lenses.

Items of interest may be inclusive of a significantly large part of the medical terminology model leading to obvious problems of scale. While normally presenting the mapper's results on the lens is beneficial, sometimes it is not necessary and some form of indication of the result is sufficient. A simple solution would be to allow a user to specify a threshold to prevent overloading the lenses with too much information. However, this should be further investigated.

Performance The performance of the lens is currently disappointing. Queries to $\mathrm{TeS}$ takes a significant amount of time to answer and queries are formed by the lenses faster than they can be answered. Figure 4 took around 10 seconds to obtain all the answers. Currently, to minimize the amount of computation, a lens update only occur upon a mouse button release, after a movement on the surface, or new objects has been created. An obvious feature to provide to minimise the query-to-answer delay, is the caching of results from the TeS. Lenses like Mapper and Visualizer are also reducing the interactive performance of PadDraw, due to the low rendering speed of a large number of result items.

\section{CONCLUSION AND FUTURE WORK}

This paper has presented a suite of techniques for inspecting GRAIL concept models based on the Movable Lens interface paradigm. These techniques enable modelers to inspect concept models during their authoring in a variety of flexible ways, and provide facilities for managing re-usable parts. 
Instead of being locked within loops of select, display and "comprehension by reading", each of limited bandwidth and scope, users have a much larger freedom of choice. The features of the interface are summarized as follows:

- Ability to construct queries and visualizations compositionally and progressively by directly manipulating stacks of lenses. Multiple linkages can be achieved with the use of portals.

- The information at any step can be visualized, manipulated and selected using lenses in a variety of ways. Different questions about sub-parts of model can be asked simultaneously and results compared graphically with overlapping lenses.

- The movable lens stacks allow fast switching between different region of interests. Previous query results are hidden once the lens is removed, but can be reconstructed by relocating the lens.

- The environment is extensible and provides an uniform method to integrate new forms of lenses, visual representations and queries. A node-link diagram generator could be included in a Visualizer lens for example.

The choice of PadDraw and Pad++ was not arbitrary. The zoomable interface offers unlimited space on which to organize the modeler's notes, useful visualization macros, as well as re-usable parts of the knowledge model. The facility of hyperlinks on the surface allows the construction of bookmarks to visualizations which can be scattered on the surface at different levels of scales.

The new tool is seen as complementary to the original text based single concept centred GRAIL browser. Once a compound of lenses with a specific macro functionality is determined to be useful, perhaps incorporating that function into the original GRAIL browser is more beneficial to modelers in general who are already familiar with it.

Our major focus now is to put it into actual use as part of the modeling suite for usability evaluation. We are also aiming to derive a variety of useful queries and visualizations extension from the usability studies.

\section{ACKNOWLEDGEMENT}

We thank Jeremy Rogers, Pam Pole, Sean Bechhofer, Danny Soloman, Conrad Toft, Patricia Baker, Veronique Lattes, and Joe Bullock for painstakingly clarifying their modeling problems, as well as their enthusiasm and encouragement in supporting the use of movable lens filters as visualization modules.

\section{REFERENCES}

Ahlberg, C., Williamson, C. \& Shneiderman, B. (1992), Dynamic Queries for Information Exploration: an Implementation and Evaluation, in 'Proceedings of CHI'92', pp. 619-626. 
Ahlberg, C. \& Wistrand, E. (1995), IVEE: An Information Visualization and Exploration Environment, in 'Proceedings of the Symposium on Information Visualization'95', pp. 66-73.

Baader, F., Jensfeld, M. \& Nutt, W., eds (1997), Proceedings of the 4th Workship Knowledge Representation meets Databases KRDB' '97, Athens.

Bechhofer, S., Goble, C. A., Rector, A., Solomon, W. \& Nowlan, W. (1997), Terminologies and Terminology Servers for Information Environments, in 'Proceedings of STEP 97, 8th International Workshop on Software Technology and Engineering Practice', London.

Bederson, B. B., Hollan, J. D., Perlin, K., Meyer, J., Bacon, D. \& Furnas, G. (1996), 'Pad++: A Zoomable Graphical Sketchpad for Exploring Alternate Interface Physics', Journal of Visual Languages and Computing 7, 3-31.

Bertin, J. (1981), Graphics and Graphic Information Processing, Walter de Gruyter and Co., Berlin.

Bier, E. A., Stone, M. C., Pier, K., Buxton, W. \& DeRose, T. D. (1993), Toolglass and Magic Lenses: The See-Through Interface, in 'Proceedings of SIGGRAPH'93', pp. 73-80.

Bier, E. A., Stone, M. C., Fishkin, K., Buxton, W. \& Baudel, T. (1994), A Taxonomy of See-Through Tools, in 'Proceedings of CHI'94', Boston, MA, pp. 358364.

Borgida, A., Brachman, R. J., McGuinness, D. L. \& Resnick, L. A. (1989), 'CLASSIC: A structural data model for objects', SIGMOD Record 18(2), 58-67.

Borgida, A. (1995), 'Description Logics in Data Management', IEEE Transaction on Knowledge and Data Engineering 7(5), 671-682.

Brodlie, K. W., Gallop, J. R., Grant, A. J., Haswell, J., Hewitt, W. T.and Larkin, S., Lilley, C. C., Morphet, H., Townend, A., Wood, J. \& Wright, H. (1995), Review of Visualization Systems (2nd Edition), Technical Report 9, Advisory Group on Computer Graphics. http://www.agocg.ac.uk/agocg/TechReports/ VisSyst/dogbook_1.html.

Devanbu, P., Brachman, R., Selfridge, P. \& Ballard, B. (1991), 'LaSSIE: A Knowledge-based Sofware Information System', Communication of $A C M$ 34(5).

Dodson, D., Secker, J., Scott, R. \& Hugh, R. (1995), VIM: 3D Co-operative Diagrams as KBS Surfaces, in 'Proceedings of Expert Systems'95', Cambridge. http://www.soi.city.ac.uk/research/dig/95/es95/es95.ps.

Fairchild, K. M., Poltrock, S. E. \& Furnas, G. W. (1988), SemNet: ThreeDimensional Graphic Representations of Large Knowledge Bases, in R. Guindon, ed., 'Cognitive Science and its applications for Human Computer Interaction', Lawrence Erlbaum Asscociates Inc., pp. 201-233.

Fishkin, K. \& Stone, M. C. (1995), Enchanced Dynamic Queries via Movable Filters, in 'Proceedings of CHI'95'.

Keim, D. A. \& Kriegel, H.-P. (1994), 'VisDB: Database Exploration Using Multidimensional Visualization', IEEE Computer Graphics and Applications pp. $40-49$. 
Paley, S., Karp, P. \& Lowrance, J. (1996), ‘Generic Knowledge-Base Editor’, SRI International. http://www.ai.sri.com/gkb.

Patel, U. K. \& Sutcliffe, A. G. (1993), Three-Dimensional Visualisation of Knowledge Structures: Prototyping for Design Evaluation, in B. Leonard, J \& J. Gornostaev, eds, 'Proceedings of EWHCl'93', Springer Verlag, pp. 163180. LNCS 753.

Rector, A. L., Nowlan, W. A. \& Glowinski, A. J. (1993), Goals for Concept Representation in the GALEN project, in 'Proceedings of 17th Annual Symposium on Computer Applications in Medical Care, SCAMC '93', pp. 414-418.

Rector, A. L., Bechhofer, S. K., Goble, C. A., Horrocks, I., Nowlan, W. A., \& Solomon, W. D. (1997), 'The GRAIL Concept Modelling Language for Medical Terminology', Artificial Intelligence in Medicine 9, 139-171.

Rychtyckyj, N. (1996), DLMS: An Evaluation of KL-ONE in the Automobile Industry, in 'Proceedings of KR'96', pp. 588-596.

Stone, M. C., Fishkin, K. \& Bier, E. A. (1994), The Movable Filter as a User Interface Tool, in 'Proceedgins of CHI'94', Boston, MA, pp. 306-312.

Tweedie, L. A., Spence, R., Dawkes, H. \& Su, H. (1996), Externalizing Abstract Mathematical Models, in 'Proceedings of CHI'96', pp. 406-412.

\section{BIOGRAPHY}

Gary Ng is a Ph.D. student and teaching assistant in Computer Science at the University of Manchester. He received his B.Sc. in Computing and Information Systems in 1993 and M.Sc. in High Performance System Design in 1994, both at the University of Manchester. His Ph.D. research topic is "Conceptual Knowledge Visualization". His research interests include user-interfaces to databases, diagrammatic representations and data visualization.

Carole Goble is a senior lecturer in information systems at the University of Manchester, where she co-leads the Information Management Group. She was a research associate at Manchester from 1982 and joined the faculty in 1985. Her research interests include hypermedia and multimedia information systems, description logics for managing data and metadata, user-interfaces to databases, and distributed information systems.

Dr. Adrian West is a Lecturer in Computer Science, and a founder of the Advanced Interfaces Group. His B.Sc. (1982) and Ph.D. (1987) are both in Computer Science from the University of Manchester. He has worked in the automated test equipment industry, and on parallel implementations of secure networks at Logica Space and Defense. He has published and presented work on the monitoring of parallel systems and the development of high level graphical environments for parallel programming. His current research interests include VR operating system architecture, and the metaphysical structure of virtual environments. 